\title{
Using Proof-of-Work to Mitigate Spoofing-Based Denial of Service Attacks
}

\author{
Samuel DeLaughter ${ }^{\star} \dagger$ \\ samd@mit.edu \\ Massachusetts Institute of Technology \\ Cambridge, MA, United States
}

\author{
Karen R. Sollins* \\ sollins@csail.mit.edu \\ Massachusetts Institute of Technology \\ Cambridge, MA, United States
}

\begin{abstract}
This paper proposes a new strategy for using proof-of-work (PoW) to mitigate certain Denial of Service attacks which rely on address spoofing. By requiring clients to include a very small PoW in their requests to establish new connections, servers can protect connection resources from spoofers who lack the processing power to compute sufficient proofs for each packet in their floods. We present a methodology for evaluating this system empirically in a network testbed.
\end{abstract}

\section{CCS CONCEPTS}

- Networks $\rightarrow$ Denial-of-service attacks; Packet scheduling.

\section{KEYWORDS}

Denial of Service, Address Spoofing, Proof-of-Work

\section{ACM Reference Format:}

Samuel DeLaughter and Karen R. Sollins[1]. 2021. Using Proof-of-Work to Mitigate Spoofing-Based Denial of Service Attacks. In CoNEXT Student Workshop 2021 (CoNEXT-SW'21), December 7, 2021, Virtual Event, Germany. ACM, New York, NY, USA, 2 pages. https://doi.org/10.1145/3488658.3493789

\section{PROBLEM STATEMENT}

The ability to send packets with spoofed IP addresses remains a serious threat to the Internet. A malicious actor can masquerade as many different devices in order to monopolize some network resource(s), as in a TCP SYN flood or QUIC flood. While many ISPs have deployed ingress filtering to mitigate this threat, address spoofing remains possible in over $30 \%$ of all ASes, and this state of affairs has seen no real improvement in the past decade[1, 6]. As long as spoofing is possible anywhere it poses a threat to the entire Internet, and unfortunately there is no clear path to universal deployment of ingress filtering.

The standard solution is to perform source address validation via a challenge-response protocol. When a server receives a request to establish a new connection it first responds with a cryptographic token, then waits until the client echoes that token back before allocating state for the connection. A malicious client spoofing

*Funded by SystemsThatLearn@CSAIL

${ }^{\dagger}$ Funded by Defense Advanced Research Projects Agency contract HR001118C0059

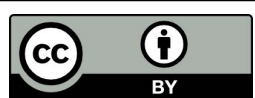

This work is licensed under a Creative Commons Attribution International 4.0 License. CoNEXT-SW'21, December 7, 2021, Virtual Event, Germany

(C) 2021 Copyright held by the owner/author(s).

ACM ISBN 978-1-4503-9133-7/21/12.

https://doi.org/10.1145/3488658.3493789 its address will be unable to receive the token, and so the server avoids wasting scarce resources. Such a system is absent from the original TCP standard but is implemented as "SYN Cookies," which are enabled by default on many modern operating systems[3]. This approach is also built into the recently standardized transport protocol QUIC in the form of a "Retry" packet[4].

These challenge-response protocols have been broadly adopted despite an absence of empirical evaluation. There is a small but non-negligible amount of overhead required to generate the token, send a response packet (eg. a SYN-ACK including a SYN Cookie or a QUIC Retry message), and verify tokens if and when they are echoed back. In preliminary experiments, we found that the overhead of SYN Cookies is so significant it can outweigh their benefits and actually degrade client quality of service rather than improving it. There is a clear need for novel spoofing mitigations that place less of a burden on the servers they intend to protect.

\section{PROPOSAL}

Though devices in certain regions can easily send packets with many different source addresses, they still only have the computational resources of a single device. We propose a new system which takes advantage of this fact by using PoW to rate-limit certain spoofing attacks with little or no overhead at the targeted server.

The general concept of using PoW to combat malicious traffic on the Internet is not new - in fact PoW originated as a defense against spam email[2]. While that particular application failed (likely due to reasons described in [5]), the network security landscape has changed a great deal in the last 30 years and modern DoS attacks have little in common with spam email. Our system not only addresses a categorically different threat from that considered in [2] and [5], it also operates in an entirely novel way:

(1) Let $\mathrm{C}$ be a client which wants to establish a connection (eg. TCP or QUIC) with some server S.

(2) C composes its initial message $m$ as normal (eg. a TCP SYN packet or QUIC Initial packet) and computes the message's hash $H(m)$ using some well-known hash function. Note that this hash encompass the entire transport-layer header as well as the source and destination IP addresses and port numbers. It must not encompass any fields which may be altered in transit.

(3) C then increments some field of the message that was set randomly in the message's initial construction. Suitable fields include the Sequence Number and/or Acknowledgement Number Fields in TCP, and the Source/Destination Connection ID fields in QUIC. We refer to whichever field is incremented as the "nonce". 
(4) $\mathrm{C}$ repeats the previous step $k$ times, keeping track of the nonce value that produces a message with the highest hash value, $\bar{m}$. The value $k$ here is a security parameter that can be tuned depending on the perceived threat and the client's computational resources.

(5) C sends $\bar{m}$ towards S.

(6) S, or some middlebox $M$ on the path from $C$ to $S$ receives $\bar{m}$ and computes its hash.

(7) S/M then implements one of the following traffic shaping strategies (we will evaluate both but expect them to produce similar results):

(a) Append the message to one of multiple drop-tail queues, serviced according to a weighted round-robin algorithm with weights based on their hash values, such that queues containing messages with higher hashes are serviced more frequently.

(b) The hash is compared against some threshold based on the receiver's current load. If the hash falls below the threshold the packet is dropped. Otherwise (or if the receiver is under low load) the message is processed normally.

\section{HYPOTHESES}

We expect this system to outperform traditional challenge-response schemes (like SYN Cookies and QUIC Retry packets) for several reasons. First, our method offloads much of the burden of verification from the server being defended, requiring it to compute at most a single hash. Better yet, it can be deployed as a middlebox solution which eliminates all mitigation overhead at the server. Additionally, it operates entirely within the first packet, whereas sending TCP SYN-ACKs or QUIC Retries to spoofed addresses only adds more unwanted traffic to an already congested network.

Finally, our system is unique in that it handles packets based on the expected amount of work expended to create them, rather than requiring a single correct answer. This allows us to seamlessly accommodate legacy devices, as they are equivalent to a participating device with $k=1$. Non-participating devices should experience no significant change in service quality outside periods of attack or otherwise excessive demand.

The key trade-off in this system is that clients will experience slight increases in latency and CPU utilization when sending connection requests. The question is how much overhead is required to measurably increase DoS resilience. As detailed in [5], this is the main reason PoW failed in the context of spam email mitigation the economics of that trade-off favored attackers.

Our system leverages an inherent asymmetry between the behavior of spoofers and legitimate clients. The former may send tens of thousands of connection requests per second, while the latter typically send just one to initiate a long-running session. This disparity will enable clients to significantly "outbid" attackers by devoting just a small fraction of their CPU time to PoW. We hypothesize that measurable increases in DoS resilience will be attainable with latency overhead on the order of $1 \mathrm{~ms}$ per initial packet.

Note that this system only defends against spoofers who masquerade as many different devices. It does not protect against reflection attacks or large-scale botnets.

\section{EXPERIMENTS}

We plan to test this system through experimentation on the DeterLab network testbed, which has been specifically designed for both realistic performance and the effective sandboxing of malicious traffic. We will deploy a server which listens for TCP/QUIC connections, a set of clients which attempt to establish connections with the server as quickly as possible, and a set of attackers who attempt to monopolize the server's resources by sending a flood of connection requests with spoofed source addresses.

After taking baseline measurements, we will implement our system at different locations in the network: on the server, as a middlebox near the server, and as multiple middleboxes at the network edge. In each case we will measure the system's impact on client QoS. We plan to follow best practices established by Mirkovic et. al. for evaluating QoS [7], with our primary metric being the time required to establish a new connection with the server, since its ability to accept new connection requests is the critical resource our system is designed to protect. Fine-grained measurements of CPU and memory utilization at the clients, servers, and middleboxes will be used to evaluate the system's overhead.

We will also experiment with different values of $k$ and different rates of attack, in order to determine how much time clients must spend on PoW computation in order to keep spoofers below a given rate limit. Finding an optimal $k$ value is difficult, since it requires weighing a small constant decrease in QoS against an occasional large increase. We hope to see significant benefits with $<1 \mathrm{~ms}$ of latency overhead on legitimate clients.

\section{RESULTS}

The results and precise methodology of our experiments will be written up and submitted for peer review upon completion of this work. We will also make our code and collected data available in order to facilitate replication studies and/or future work in this vein. Assuming our results conform to expectation and our system succeeds in mitigating spoofing attacks, we will work through the IETF to standardize it for real-world deployment.

\section{REFERENCES}

[1] Robert Beverly, Arthur Berger, Young Hyun, and k claffy. 2009. Understanding the Efficacy of Deployed Internet Source Address Validation Filtering. In Proceedings of the 9th ACM SIGCOMM Conference on Internet Measurement (IMC '09). ACM, New York, NY, USA, 356-369. https://doi.org/10.1145/1644893.1644936

[2] Cynthia Dwork and Moni Naor. 1993. Pricing via Processing or Combatting Junk Mail. In Advances in Cryptology - CRYPTO' 92, Ernest F. Brickell (Ed.). Vol. 740. Springer Berlin Heidelberg, Berlin, Heidelberg, 139-147. https://doi.org/10.1007/3540-48071-4_10 Series Title: Lecture Notes in Computer Science.

[3] Wesley M. Eddy. 2007. TCP SYN flooding attacks and common mitigations. RFC 4987. Internet Engineering Task Force. http://tools.ietf.org/html/rfc4987.txt

[4] Jana Iyengar and Martin Thomson. 2021. QUIC: A UDP-Based Multiplexed and Secure Transport. Request for Comments RFC 9000. Internet Engineering Task Force. https://doi.org/10.17487/RFC9000 Num Pages: 151.

[5] Ben Laurie and Richard Clayton. 2004. Proof-of-work proves not to work. In Workshop on Economics and Information Security, Vol. 2004.

[6] Matthew Luckie, Robert Beverly, Ryan Koga, Ken Keys, Joshua A. Kroll, and k claffy. 2019. Network Hygiene, Incentives, and Regulation: Deployment of Source Address Validation in the Internet. In Proceedings of the 2019 ACM SIGSAC Conference on Computer and Communications Security. ACM, London United Kingdom, 465-480. https://doi.org/10.1145/3319535.3354232

[7] Jelena Mirkovic, Alefiya Hussain, Sonia Fahmy, Peter Reiher, and Roshan K. Thomas. 2009. Accurately Measuring Denial of Service in Simulation and Testbed Experiments. IEEE Transactions on Dependable and Secure Computing 6, 2 (April 2009), 81-95. https://doi.org/10.1109/TDSC.2008.73 